Oncology

\title{
Perioperative management of hypertensive neuroblastoma: A study from the Italian Group of Pediatric Surgical Oncologists (GICOP)
}

\author{
Luca Pio $^{\mathrm{a}, \mathrm{b}, *}$, Stefano Avanzini a , Girolamo Mattioli a,b, Giuseppe Martucciello a,b ${ }^{\mathrm{a}}$, Angela Rita Sementa ${ }^{\mathrm{c}}$, \\ Massimo Conte ${ }^{\mathrm{d}}$, Annarita Gigliotti ${ }^{\mathrm{e}}$, Claudio Granata ${ }^{\mathrm{f}}$, Ernesto Leva ${ }^{\mathrm{g}}$, Anna Maria Fagnani ${ }^{\mathrm{g}}$, \\ Umberto Caccioppoli ${ }^{\mathrm{h}}$, Nino Tedesco ${ }^{\mathrm{h}}$, Jurgen Schleef ${ }^{\mathrm{i}}$, Elisa Tirtei ${ }^{\mathrm{j}}$, Fortunato Siracusa ${ }^{\mathrm{k}}$, Paolo D'Angelo ${ }^{1}$, \\ Pierluigi Lelli Chiesa ${ }^{\mathrm{m}}$, Lucia Miglionico ${ }^{\mathrm{n}}$, Bruno Noccioli ${ }^{\mathrm{o}}$, Elisa Severi ${ }^{\mathrm{o}}$, Claudio Carlini ${ }^{\mathrm{p}}$, Francesco Vaccarella ${ }^{\mathrm{p}}$, \\ Francesco Camoglio ${ }^{\mathrm{q}}$, Simone Cesaro ${ }^{\mathrm{r}}$, Alessandra Narciso ${ }^{\mathrm{s}}$, Giovanna Riccipetitoni ${ }^{\mathrm{t}}$, \\ Giovanni Cecchetto ${ }^{\mathrm{u}}$, Alessandro Inserra ${ }^{\mathrm{s}}$
}

a Pediatric Surgery Unit, Istituto Giannina Gaslini, Genova

b DINOGMI, Università Degli Studi di Genova

c Pathology Unit, Istituto Giannina Gaslini, Genova

d Oncology Unit, Istituto Giannina Gaslini, Genova

e Epidemiology Biostatistics and Committees Unit, Istituto Giannina Gaslini, Genova

${ }^{\mathrm{f}}$ Radiology Unit, Istituto Giannina Gaslini, Genova

g Pediatric Surgery Unit, Fondazione IRCCS Ca' Granda Ospedale Maggiore Policlinico, Milano

${ }^{\text {h }}$ Surgical Oncology Unit, A.O.R.N. Santobono Pausilipon, Napoli

i Pediatric Surgery Unit, Ospedale Infantile Regina Margherita, Torino

${ }^{\mathrm{j}}$ Oncology Unit, Ospedale Infantile Regina Margherita, Torino

${ }^{\mathrm{k}}$ Policlinico Universitario P. Giaccone, Palermo

${ }^{1}$ Oncology Unit, A.R.N.A.S. Civico, Di Cristina and Benfratelli Hospital, Palermo

m Pediatric Surgery Unit, Ospedale Santo Spirito, Pescara

n Oncology Unit, Casa Sollievo della Sofferenza, San Giovanni Rotondo

${ }^{\circ}$ Pediatric Surgery Unit, Ospedale Pediatrico Meyer, Firenze

P Pediatric Surgery Unit, ASN SS: Antonio e Biagio e Cesare Arrigo, Alessandria

${ }^{q}$ Pediatric Surgery Unit, Azienda Ospedaliera Universitaria Integrata di Verona, Verona

${ }^{\mathrm{r}}$ Oncology Unit, Azienda Ospedaliera Universitaria Integrata di Verona, Verona

${ }^{s}$ Pediatric Surgery Unit, Ospedale Pediatrico Bambino Gesù, Roma

${ }^{\mathrm{t}}$ Pediatric Surgery Unit, Ospedale Pediatrico Buzzi, Milano

u Azienda Ospedaliero-Universitaria, Padova

\section{A R T I C L E I N F O}

Article history:

Received 23 October 2016

Received in revised form 13 June 2017

Accepted 29 June 2017

\section{Key words:}

Neuroblastoma

Hypertension

IDRFs

Pediatric surgical oncology

Risk factors

\begin{abstract}
A B S T R A C T
Background: Hypertension (HT) is rarely reported in patients affected by Neuroblastoma (NB), and management guidelines are lacking. Clinical features and perioperative medical treatment in such patients were reviewed to 1) ascertain whether a shared treatment strategy exists among centers and 2) if possible, propose some recommendations for the perioperative management of HT in NB patients.

Methods: A retrospective multicenter survey was conducted on patients affected by NB who presented HT symptoms. Results: From 2006 to 2014, 1126 children were registered in the Italian Registry of Neuroblastoma (RINB). Of these, 21 with HT (1.8\%) were included in our analysis. Pre- and intraoperative HT management was somewhat dissimilar among the participating centers, apart from a certain consistency in the intraoperative use of the alpha- 1 blocker urapidil. Six of the 21 patients (28\%) needed persistent antihypertensive treatment at a median follow-up of 36 months (range 496 months) despite tumor removal. Involvement of the renal pedicle was the only risk factor constantly associated to HT persistency following surgery. A correlation between the presence of HT and the secretion of specific catecholamines and/or compression of the renal vascular pedicle could not be demonstrated.
\end{abstract}

Abbreviations: NB, neuroblastoma; HT, hypertension; RINB, Italian Registry of Neuroblastoma; GICOP, Italian Group of Pediatric Surgical Oncologists; IDRFs, image-defined risk factors;

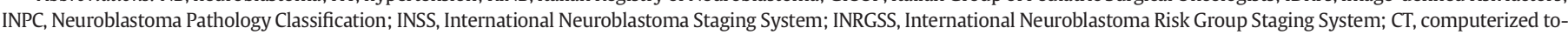
mography; MRI, magnetic resonance imaging; VMA, vanillylmandelic acid; HVA, homovanillic acid.

* Corresponding author at: Pediatric Surgery Unit, Istituto Giannina Gaslini, Largo G. Gaslini 5, 16147, Genoa, Italy. Tel.: + 3901056362217 ; fax: + 390103075092.

E-mail address: lucakeats@hotmail.it (L. Pio). 
Conclusion: Based on this retrospective review of NB patients with HT, no definite therapeutic protocol can be recommended owing to heterogeneity of adopted treatments in different centers. A proposal of perioperative HT management in NB patients is however presented.

Level of evidence: IV.

Neuroblastoma (NB) is one of the most frequent pediatric solid tumors. It often originates from the adrenal gland or from the adjacent paravertebral sympathetic ganglia and frequently involves the kidney.

Hypertension (HT) is a relatively rare symptom of NB, its reported incidence ranging between $10 \%$ and $19 \%$ of cases. Few studies have focused on the perioperative management of HT, and most of these have been case reports [1-9]. Both cardiac and neurological complications of HT have been described [10].

Both catecholamine secretion and renal vascular compression have been advocated as the possible pathophysiological mechanism of HT in NB $[2,3]$. Although the secretion of catecholamines may be increased in NB patients, HT and other symptoms of catecholamine excess frequently observed in pheochromocytoma have been reported in relatively few children with NB (1-9). This difference is probably owing to the presence of many storage granules in pheochromocytoma, which release large quantities of catecholamines, thereby causing HT. In neuroblastic cells, this storage mechanism seems to be inefficient, causing intracellular granule disruption, the inactivation of catecholamines and the release of their metabolites into the circulation. A small number of NB do display abundant storage granules and patients can exhibit blood pressure patterns like those of patients with pheochromocytoma [11-14]. Chemotherapy may result in additional catecholamine release and HT owing to tumor lysis, thus warranting close observation during preoperative cycles [4].

Renal artery stenosis is a possible cause of HT and has been observed in about $3 \%$ to $10 \%$ of cases of pediatric $\mathrm{HT}[15,16]$. NB causing renovascular HT owing to extrinsic aortic narrowing and/or stenosis of the renal artery has been reported $[2,5]$.

Preoperative echocardiography is the cornerstone in the diagnostic workup of these patients, as catecholamine excess can induce several complications other than, or subsequent to, HT, including seizures and heart failure, cardiomyopathy, cardiomegaly and cardiogenic shock [3,6,15,17-26]. All in all, preoperative HT control has lowered reported mortality from $13 \%$ to $45 \%$ to $0 \%-3 \%$ in patients with catecholaminesecreting tumors [27].

The anesthesiological intraoperative management of hyper- and hypotensive peaks has rarely been reported [3,4]. Whatever the cause of HT in $\mathrm{NB}$, symptoms are generally well controlled by the preoperative administration of alpha- 1 adrenergic blocking agents, such as phenoxybenzamine, phentolamine, prazosin and, more recently, doxazosin [3,4,6,9,17-26].

Despite effective preoperative alpha-1adrenergic blockade, intraoperative HT may still occur, especially when the tumor is being manipulated. Therefore, the availability of a wide range of rapidly acting medications to treat intraoperative hypertensive peaks is mandatory; these include prostaglandin E1, diuretics, calcium channel antagonists, sodium nitroprusside, hydralazine, magnesium sulfate, phentolamine, adenosine, and fenoldopam [4,6,20-22]. Beta blockers, such as propranolol, labetalol, atenolol and esmolol, may be administered intraoperatively in association with alpha-1 blockers to control catecholamineinduced arrhythmia and tachycardia [4,6]. Postresection hypotension may be successfully managed by means of continuous iv administration of dopamine in the first 24 postoperative hours [6].

In the present study, we retrospectively reviewed an Italian series of NB patients with HT, with a view to assessing the possible correlation between HT and the secretion of specific catecholamines and/or compression of the renal vascular pedicle. We also evaluated the perioperative management of HT to find out whether a possible treatment recommendation can be presented.

\section{Materials and methods}

The Italian Registry of Neuroblastoma (RINB) collects all newly diagnosed cases of NB in Italy, together with demographic, chemotherapy, surgical, and follow-up data.

The Italian Group of Pediatric Surgical Oncologists (GICOP) conducted a retrospective survey of all the patients affected by NB with associated HT registered in the RINB from 2006 to 2014. HT was defined as blood pressure values higher than the 99th percentile $+5 \mathrm{mmHg}$, as stated by the National High Blood Pressure Education Program Working Group in 2004 [28]. All children who had undergone surgical resection were included in the present study.

The following data were retrospectively collected in an electronic database: series demographics, diagnostic workup, including assays of urinary and/or blood catecholamines, image-defined risk factors (IDRFs) present on preoperative imaging (CT or MRI), tumor histology according to the International Neuroblastoma Pathology Classification (INPC) [29], tumor staging according to the International Neuroblastoma Staging System (INSS) [30] and the International Neuroblastoma Risk Group Staging System (INRGSS) [31], and biomolecular features such as the presence or absence of MYCN amplification.

Pre-, peri- and postoperative antihypertensive treatment regimens were examined. Treatment duration, drugs administered and possible HT persistence during follow-up were recorded.

Anesthesiological treatments were collected and divided into premedication, epidural, induction, muscle relaxation, maintenance and the need for packed red blood cell transfusion.

Surgery was defined as complete if more than $95 \%$ of the tumor had been resected (microscopic residual disease), as leaving minimal residual disease (if more than $90 \%$ but less than $95 \%$ of the tumor had been removed, macroscopic minimal residual disease), or as incomplete (if less than $90 \%$ of the tumor had been removed).

Clinical follow-up examination was conducted by a pediatric nephrologist, an oncologist and a cardiologist, and included 24-h continuous blood pressure measurement and radiological reevaluation (CT, MRI and ultrasonography scan).

\section{Results}

From 2006 to 2014, 1126 children affected by NB were registered in the RINB, 25 of whom presented HT (2.2\%). Four of these 25 children did not undergo surgical resection, and were therefore excluded from the analysis. Consequently, the study cohort comprised 21 patients. Patients' demographics and tumor characteristics are detailed in Table 1.

Vanillylmandelic acid (VMA) and homovanillic acid (HVA) were tested in all patients and were found elevated in 9 (43\%).Norepinephrine and epinephrine were assayed in only 3 patients, and proved to be elevated in all of them. Dopamine was never analyzed.

An age-comparable series of 28 patients presenting with non-HT NB and involvement of the renal vascular pedicle was examined to detect any significant differences. The only finding that emerged from this analysis was that a relatively higher proportion of patients in the nonhypertensive group displayed increased VMA/HVA (89\% of normal-tension NB patients vs $43 \%$ of HT NB patients). No statistical analysis was conducted on these data. 
Table 1

Clinical characteristics of the study patients $(\mathrm{N}=21)$.

\begin{tabular}{|c|c|c|}
\hline Clinical characteristics & N./Total & $\%$ \\
\hline \multicolumn{3}{|l|}{ Gender: } \\
\hline - Male & 11 & $52.3 \%$ \\
\hline - Female & 10 & $47.6 \%$ \\
\hline \multirow[t]{2}{*}{ Age at surgery (months), median (range) } & 15 & \\
\hline & \multicolumn{2}{|c|}{ (1 month-18 years) } \\
\hline \multicolumn{3}{|l|}{ Stage INSS (INRGSS) } \\
\hline$\cdot 1(\mathrm{~L} 1)$ & 5 & $23.8 \%$ \\
\hline - 3 (L2) & 9 & $42.9 \%$ \\
\hline - $4(\mathrm{M})$ & 7 & $33.3 \%$ \\
\hline Renal pedicle involvement (IDRF) & 8 & $38 \%$ \\
\hline \multicolumn{3}{|l|}{ Histology: } \\
\hline - Schwannian stoma poor Neuroblastoma & 20 & $95.2 \%$ \\
\hline - Ganglioneuroblastoma Intermixed & 1 & $4.8 \%$ \\
\hline \multicolumn{3}{|l|}{ MYCN status: } \\
\hline - Amplified & 1 & $4.8 \%$ \\
\hline - Not Amplified & 20 & $95.2 \%$ \\
\hline \multicolumn{3}{|l|}{ Vanillylmandelic acid (VMA) and homovanillic acid (HVA) } \\
\hline - Normal values & 11 & $57 \%$ \\
\hline - Elevated values & 9 & $43 \%$ \\
\hline Preoperative chemotherapy & 12 & $57.1 \%$ \\
\hline Preoperative antihypertensive treatment & 18 & $85.7 \%$ \\
\hline $\begin{array}{l}\text { Preoperative antihypertensive treatment duration (days), } \\
\text { median (range) }\end{array}$ & \multicolumn{2}{|c|}{$90(5-300)$} \\
\hline Persistent postoperative HT & 6 & $28.6 \%$ \\
\hline \multicolumn{3}{|l|}{ Surgical resection: } \\
\hline - Complete & 15 & $71.4 \%$ \\
\hline - Minimal Residual & 2 & $9.6 \%$ \\
\hline - Incomplete & 4 & $19 \%$ \\
\hline \multicolumn{3}{|l|}{ Follow-up (median 36 months, range 4-96 months): } \\
\hline - Complete remission & 12 & $57.1 \%$ \\
\hline - Residual disease & 6 & $28.6 \%$ \\
\hline - Progression of disease/death & 3 & $14.3 \%$ \\
\hline
\end{tabular}

Preoperative radiological imaging showed normal-sized kidneys (ageand sex-matched) in all patients. Involvement of the renal vascular pedicle was observed in 8 of the 21 patients (38\%) in our study cohort (Fig. 1).

The four excluded patients had not undergone surgery owing to the persistence of 3 or more IDRFs after chemotherapy (Patient 1: bilateral renal pedicle, aorta, celiac artery, superior mesenteric artery; Patient 2: renal pedicle, hepatic pedicle, cava, aorta; Patient 3: renal pedicle, superior mesenteric artery, aorta; Patient 4: renal pedicle, cava, aorta). Only Patient 4 had increased VMA/HVA. Patients 1 and 2 are alive with active disease and are on dual anti-HT therapy. Patients 3 and 4 have died.

None of the patients presented with cardiac hypertensive-related complications on preoperative echocardiography.

Preoperative antihypertensive treatment was necessary in 18 patients (86\%) for a median preoperative time of 90 days (range: 5-300 days). This consisted of single-drug therapy in 8 patients and multiple-drug therapy in 10 patients (Table 2 ).

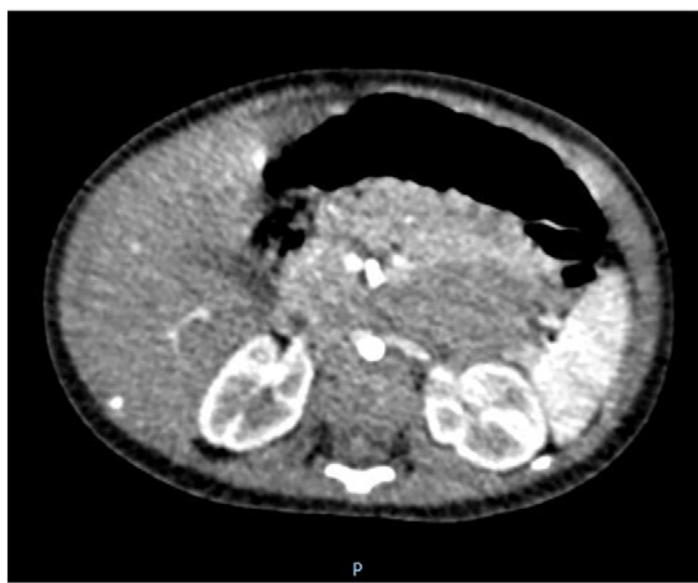

Fig. 1. Computerized tomography (axial section): encasement of renal pedicles.
Table 2

Characteristics of the antihypertensive treatment in 21 patients.

\begin{tabular}{llll}
\hline & Hypertensive treatment & $\mathrm{N}$ & $\%$ \\
& & patients & patients \\
\hline Preoperative & & 18 & 85.7 \\
Single-drug treatment & 3 & \\
& ACE inhibitors (captopril) & 3 & \\
& Calcium antagonist (amlodipine) & 2 & \\
Multiple-drug & Beta blockers (atenolol) & 10 & \\
treatment & 1. Beta blockers (atenolol) & 9 & \\
(2-4 drugs) & $\quad$ + Calcium antagonists & \\
& (nifedipine) & 5 \\
& $\quad$ + Alpha blockers (doxazosin) & 4 & \\
Intraoperative & $\quad$ ACE inhibitors (captopril) & 3 & \\
Single-drug treatment & Alpha Blockers (urapidil) & 6 & 28.6 \\
Double-drug treatment & 1. Diuretics (furosemide) & 1 & \\
& 2. Calcium antagonists (nifedipine) & 1 & \\
Postoperative & & 6 & 28.6 \\
Single-drug treatment & Calcium antagonists (nifedipine) & 2 & \\
Double-drug treatment & 1. Calcium antagonists (nifedipine) & 4 & \\
& 2. Beta Blockers (atenolol) & 4 & \\
\hline
\end{tabular}

The induction and maintenance of anesthesia were almost uniform in all centers, with midazolam being used in 19 patients (90\%), propofol for the induction phase in 17 (81\%), rocuronium for muscle relaxation in $18(86 \%)$ and sevorane for anesthesia maintenance in all patients. Epidural anesthesia was performed in 9 patients (43\%). Six patients developed intraoperative hypertensive peaks, which were successfully manager with alpha 1 blockers (urapidil) in 5 cases and with a combination of calcium antagonist (nifedipine) plus diuretic (furosemide) in the remaining case.

No hypertensive complications were documented during surgical procedures.

Surgical results are detailed in Table 1 . None of the patients in this series underwent nephrectomy to achieve complete resection.

On follow-up examination conducted after a median of 36 months (range 4-96 months), 12 patients were in complete remission (57\%), 6 presented with residual disease (28\%) and 3 had died owing to disease progression (14\%). Six patients (28\%) presented persistent postoperative HT despite complete tumor resection in 4 of them and minimal residual in 1. Long-term antihypertensive treatment was therefore started in these patients. Four of these six patients had been treated with antihypertensive medications for more than 100 days preoperatively.

Six out of 8 children with documented involvement of the renal pedicle (on IDRF evaluation) presented persistence of HT after surgery. As these 6 patients constituted the whole cohort of children with persistent postoperative HT, renal pedicle involvement seems to be the only risk factor predictive of persistent HT (owing to the small number, no statistical analysis was conducted on these data). These patients underwent a thorough postoperative radiological assessment by means of US-Doppler and CT scan to detect possible renal hypotrophy/atrophy possibly owing to persistent spasm of the renal artery owing to prolonged intraoperative vessel manipulation and/or impaired renal perfusion observed during surgery. This analysis revealed no pathological findings in patients with persistent HT, showing normal-shaped and normal-sized kidneys in all of them, regardless of the involvement of the renal pedicle.

\section{Discussion}

NB is one of the most common solid tumors in infancy, accounting for approximately $8 \%$ of childhood cancers. HT was documented in nearly $2.2 \%$ of the cases recorded in the RINB, thus constituting a rare condition associated to NB; its management varies widely according to the center involved.

To the best of our knowledge, the present study analyzed the largest series of children affected by NB with HT. Nevertheless, it is still limited, 
and no definite conclusions can be drawn owing to the heterogeneity of diagnostic and therapeutic protocols within the different centers involved in the study. Unfortunately, only urinary metabolites of vasoactive amines, namely VMA and HVA, were routinely investigated in all patients, and few centers performed detailed assays of dopamine, epinephrine and norepinephrine.

Surprisingly, only 1 patient from our series showed MYC-N amplification. We could not find any clinical explanation for such an unusual distribution (considering that at least 16 patients had stage L2/M disease).

Although none of the patients from our series presented with cardiac hypertensive-related complications, a complete cardiologic assessment, including echocardiography, is mandatory in the correct preoperative workup of these patients [3,6,11,25,26].

With regard to the antihypertensive medications used in our series, the wide heterogeneity in drug selection, both preoperatively and perioperatively, is immediately evident. This is mainly owing to the lack of a recognized and accepted guideline dedicated to cases of NB presenting with HT. However, a certain consistency was observed regarding the use of one rapidly acting alpha- 1 blocker, namely urapidil, which proved to be quite effective in the management of intraoperative hypertensive peaks. Although described in the intraoperative management of pheochromocytoma in adult patients [23], to the best of our knowledge, urapidil administration has never been reported in children affected by NB presenting with HT.

In this retrospective study, preoperative anti-HT therapy was not homogeneous: $18 / 21$ underwent preoperative anti-HT therapy; the remaining three patients were stage $\mathrm{L} 1$ on diagnosis and did not receive any anti-HT therapy. The three centers concerned were therefore contacted; it transpired that preoperative treatment had not been undertaken owing to the short time ( $<7$ days) between the diagnosis of hypertension and surgical intervention. In all 18 patients, preoperative anti-HT therapy achieved HT control without any clinical complication.

One patient underwent prolonged treatment (300 days) owing to the 'unresectable' nature of the tumor (stage L2 with multiple IDRFs). The patient was eventually transferred to another center and underwent surgery. As reported by Avanzini and colleagues [32], IDRFs remain unchanged in $50 \%$ of cases after chemotherapy, and this can delay surgical resection.

Nearly $30 \%$ (6/21) of the patients in our series showed persistently increased blood pressure levels following medical and surgical treatment. Of note, in only 1 of these 6 patients might this condition have been explained by persistent disease owing to incomplete surgery. We could not find any plausible reason for HT persistence in the remaining 5 patients, since all of them underwent thorough postoperative radiological assessment by means of US-Doppler and CT scan, without any renal hypotrophy/atrophy being documented. Similarly, the persistence of HT despite surgical treatment was documented in 1 out of 12 cases reported by Madre et al. [1], without any evident clinical reason.

Involvement of the renal pedicle, as detected on preoperative radiological assessment (IDRFs), seems to be the only risk factor that strongly predicts the postoperative persistence of $\mathrm{HT}$; this scenario was observed in $75 \%$ of patients with renal pedicle involvement, who constituted the whole cohort of children with persistent postoperative HT.

On the basis of this retrospective review of NB patients with HT, no definite therapeutic protocol can be recommended. However, we would highlight a certain agreement on the intraoperative use of an alpha-1 adrenergic blocker (namely urapidil) in association with a beta-adrenergic blocker and a calcium channel antagonist to control tachycardia and possible arrhythmias. An effective combination of these proposed medications may limit the risk of cardiac complications, which may affect anesthetic and surgical outcomes.

Similarly, postresection hypotension and hypovolemia should be carefully addressed by means of continuous infusion of dopamine and fluid replacement, preferably in a pediatric intensive care setting, at least for the first $24 \mathrm{~h}$ postoperatively.

This study was presented at the 48th Congress of the International Society of Pediatric Oncology (SIOP).

\section{Acknowledgment}

The authors sincerely thank Mr. Bernard Patrick for revising the text.

\section{References}

[1] Madre C, Orbach D, Baudouin V, et al. Hypertension in childhood cancer: frequent complication of certain tumor sites. J Pediatr Hematol Oncol 2006;28(10):659-64.

[2] Weinblatt ME, Heisel MA, Siegel E. Hypertension in children with neurogenic tumors. Pediatrics 1983;71(6):947-51.

[3] Hernandez MR, Shamberger RC, Seefelder C. Catecholamine-secreting neuroblastoma in a 4-month-old infant: perioperative management. J Clin Anesth 2009;21(1):54-6.

[4] Seefelder C, Sparks JW, Chirnomas D, et al. Perioperative management of a child with severe hypertension from a catecholamine secreting neuroblastoma. Paediatr Anaesth 2005;15(7):606-10.

[5] Shinohara M, Shitara T, Hatakeyama SI, et al. An infant with systemic hypertension, renal artery stenosis, and neuroblastoma. J Pediatr Surg 2004;39(1):103-6.

[6] Sendo D, Katsuura M, Akiba K, et al. Severe hypertension and cardiac failure associated with neuroblastoma: a case report. J Pediatr Surg 1996;31(12):1688-90.

[7] Fujishiro J, Sugiyama M, Ishimaru T, et al. Cyclic fluctuation of blood pressure in neonatal neuroblastoma. Pediatr Int 2014;56(6):934-7.

[8] Wang X, Liang L, Jiang Y. Nine cases of childhood adrenal tumour presenting with hypertension and a review of the literature. Acta Paediatr 2007;96(6):930-4.

[9] Haberkern CM, Coles PG, Morray JP, et al. Intraoperative hypertension during surgical excision of neuroblastoma. Case report and review of 20 years' experience. Anesth Analg 1992;75:854-8.

[10] Kwok SY, Cheng FW, Lo AF, et al. variants of cardiomyopathy and hypertension in neuroblastoma. J Pediatr Hematol Oncol 2014;36(3):e158-61.

[11] Leckie BJ, Carachi R, Wheldon T, et al. Plasma renin levels in patients with thoracic nephroblastoma. J Pediatr Surg 1989;24:601-3.

[12] Kedar A, Glassman M, Voorhess ML, et al. Severe hypertension in a child with ganglioneuroblastoma. Cancer 1981;47:2077-80.

[13] Singh W, Ramage C, Best P, et al. Nasal neuroblastoma secreting vasopressin. Cancer 1980;45:961-6.

[14] Laug WE, Siegel SE, Shaw KN, et al. Initial urinary catecholamine metabolite concentrations and prognosis in neuroblastoma. Pediatrics 1978;62:77-83.

[15] Meyers KE, Cahill AM, Sethna C. Interventions for pediatric renovascular hypertension. Curr Hypertens Rep 2014;16:422.

[16] Humbert J, Roussey-Kesler G, Guerin P, et al. Diagnostic and medical strategy for renovascular hypertension: report from a monocentric pediatric cohort. Eur J Pediatr 2015;174:23-32.

[17] Champoux L, Gauthier M. Continuous phentolamine perfusion in the treatment of severe arterial hypertension associated with neuroblastoma. Can Anaesth Soc J 1984;31:206-9.

[18] Prys-Roberts C, Farndon JR. Efficacy and safety of doxazosin for perioperative management of patients with pheochromocytoma. World J Surg 2002;26:1037-42.

[19] Tobias JD. Preoperative blood pressure management of children with catecholaminesecreting tumors: time for a change. Paediatr Anaesth 2005;15(7): 537-40.

[20] Uchida K, Kijima K, Okuda C. Anesthetic management of a patient with norepinephrine-secreting neuroblastoma by using prostaglandin E1. Masui 1992; 41:450-4.

[21] Colson P, Ryckwaert F, Ribstein J, et al. Haemodynamic heterogeneity and treatment with the calcium channel blocker nicardipine during phaeochromocytoma surgery. Acta Anaesthesiol Scand 1998;42:1114-9.

[22] Sellden H, Kogner P, Sollevi A. Adenosine for per-operative blood pressure control in an infant with neuroblastoma. Acta Anaesthesiol Scand 1995;39:705-8.

[23] Habbe N, Ruger F, Bojunga J, et al. Urapidil in the preoperative treatment of pheochromocytomas: a safe and cost-effective method. World J Surg 2013:37(5):1141-6.

[24] Steinmetz JC. Neonatal hypertension and cardiomegaly associated with a congenital neuroblastoma. Pediatr Pathol 1989;9:577-82.

[25] Cun L, Zhe M, Xinfeng Z, et al. Fetal neuroblastoma with fetal hypertension. Ultrasound Obstet Gynecol 2008;31(1):106-7.

[26] Kato M, Hirata S, Kikuchi A, et al. Neuroblastoma presenting with dilated cardiomyopathy. Pediatr Blood Cancer 2008;50(2):391-2.

[27] Kinney MAO, Narr BJ, Warner MA. Perioperative management of pheochromocytoma. J Cardiothorac Vasc Anesth 2002;16:359-69.

[28] National High Blood Pressure Education Program Working Group on High Blood Pressure in Children and Adolescents. The fourth report on the diagnosis, evaluation, and treatment of high blood pressure in children and adolescents. Pediatrics 2004; 114:555-76.

[29] Shimada H, Ambros IM, Dehner LP, et al. Terminology and morphologic criteria of neuroblastic tumors: recommendations by the International Neuroblastoma Pathology Committee. Cancer 1999;86(2):349-63.

[30] Brodeur GM, Pritchard J, Berthold F, et al. Revisions of the international criteria for neuroblastoma diagnosis, staging, and response to treatment. J Clin Oncol 1993; 11(8):1466-77.

[31] Cohn SL, Pearson AD, London WB, et al. The International Neuroblastoma Risk Group (INRG) staging system: an INRG Task Force report. J Clin Oncol 2009; 27(2):298-303.

[32] Avanzini S, Pio L, Erminio G, et al. Image-defined risk factors in unresectable neuroblastoma: SIOPEN study on incidence, chemotherapy-induced variation, and impact on surgical outcomes. Pediatr Blood Cancer 2017 [Epub ahead of print]. 